\title{
Reasonable Inferences From Quantum Mechanics: A Response to "Quantum Misuse in Psychic Literature"
}

\author{
Bernardo Kastrup, $\mathrm{PhD}$ \\ Veldhoven, The Netherlands
}

\begin{abstract}
This invited article is a response to the paper "Quantum Misuse in Psychic Literature," by Jack A. Mroczkowski and Alexis P. Malozemoff, published in this issue of the Journal of Near-Death Studies. Whereas I sympathize with Mroczkowski's and Malozemoff's cause and goals, and I recognize the problem they attempted to tackle, I argue that their criticisms often overshot the mark and end up adding to the confusion. I address nine specific technical points that Mroczkowski and Malozemoff accused popular writers in the fields of health care and parapsychology of misunderstanding and misrepresenting. I argue that, by and large-and contrary to Mroczkowski's and Malozemoff's claims-the statements made by these writers are often reasonable and generally consistent with the current state of play in foundations of quantum mechanics.
\end{abstract}

KEY WORDS: quantum mechanics, physical realism, nonlocality, psi, holistic medicine

I appreciate this opportunity to respond to Jack A. Mroczkowski and Alexis P. Malozemoff”s (2019) article "Quantum Misuse in Psychic Literature" published in this issue of the Journal of Near-Death Studies. Let me start by acknowledging that I sympathize with Mroczkowski's

Bernardo Kastrup, $\mathrm{PhD}$, philosophy (ontology, philosophy of mind); $\mathrm{PhD}$, computer engineering (reconfigurable computing, artificial intelligence), has worked as a scientist in some of the world's foremost research laboratories, including the European Organization for Nuclear Research (CERN) and the Philips Research Laboratorieswhere the "Casimir Effect" of Quantum Field Theory was discovered. He has authored many academic papers and books on philosophy and science, being a regular contributor to Scientific American. His most recent book (2019) is The Idea of the World: A Multi-Disciplinary Argument for the Mental Nature of Reality. More information is available at www.bernardokastrup.com. Correspondence regarding this article should be sent to Dr. Kastrup at email: bernardo@bernardokastrup.com. 
and Malozemoff's-henceforth 'the authors'-cause. Few scholars would deny that Quantum Mechanics (QM) has been the subject of misuse, so it is laudable that these authors have attempted to correct at least some of it. The authors' goal of encouraging others "to avoid augmenting their discussions with improper references to physics" (p. 132) is unimpeachable and timely. There is much I agree with in their paper.

However, I believe the authors overshot the mark with their criticism. In my view, some of what they considered misuse are legitimate -if sometimes poorly worded-attempts to highlight that QM defies most people's ordinary prejudices about the nature of reality. These prejudices define what is typically considered plausible or implausible, thereby motivating many people to mistakenly dismiss important possibilities in fields such as health care and parapsychology.

Although QM has been around for nearly a century, its implications haven't yet percolated through other scientific disciplines. As a matter of fact, even within physics itself, the community of 'foundations of physics'-scholars who ponder the metaphysical implications of QMis relatively small. From this perspective, it is difficult-at least in principle - to fault attempts to bring to popular attention the degrees of natural freedom that QM may open up.

Bizarrely, popular culture is still dominated by the constraints of a naïve local-realism that QM has definitively relegated to the trash bin of history. It is thus not only legitimate, but arguably even imperative, that thought leaders play a prominent role in expanding cultural horizons in this regard. The formidable momentum behind naïve localrealism must be countered, lest people continue to live under a limiting and-most importantly—mistaken view of reality.

Although some popular writers may have worded their claims inaccurately, discussing the mind-bending implications of QM both accessibly and accurately is a formidable challenge. The authors themselves -who, unlike most of the popular writers they criticized, have the advantage of being experts in the field-have been admittedly unable to do so. They compensated for this shortcoming by adding parenthetical clarifications accessible only to experts. Yet, as far as regular readers are concerned, these parenthetical clarifications do nothing to prevent misunderstandings; they merely serve as disclaimers to safeguard the authors.

The impasse readers are left with is thus the following: On the one hand, thought leaders in fields where the implications of QM are salient must engage their public on the possibilities these implications 
open up; on the other hand, they must do it in a minimally accurate manner. There is significant tension between these two goals and no magic bullet to resolve it. The best way forward may be to engage in a critical dialogue in which scholars seek to find a balance. This is the spirit of the present response.

In the next sections, I shall comment on nine specific technical points raised by the authors.

\section{Nine Technical Points}

\section{The Physical World as Illusion}

The authors suggested that the characterization of the physical world as illusory is not justified by QM. To evaluate whether this suggestion is correct requires first an understanding of what it means to claim that the world is an illusion.

Most ordinary people would take the world to be real-as opposed to illusory-if its measurable physical properties existed independently of whether and how they are observed. An act of observation should merely disclose a self-existing physical reality, not create or define it. This presumed independence from observation-technically called 'non-contextuality'-is what underlies most people's intuition of the world's concreteness. To claim that the world is an illusion therefore means to deny non-contextuality: If the physical properties of the world actually depend on how they are observed - as opposed to existing in and by themselves - then the world is an illusion.

So what does QM say about it? Operationally, non-contextuality means that the outcome of a measurement should not depend on the way another, separate but simultaneous, measurement is performed. According to quantum theory, however, this is simply not the case. The relevant question is then whether quantum theory is correct.

Since Alain Aspect's seminal experiments (Aspect, Dalibard, \& Roger, 1982; Aspect, Grangier, \& Roger, 1981, 1982), the predictions of quantum theory in this regard have been repeatedly confirmed. The year 1998 was particularly fruitful, with two remarkable experiments performed in Switzerland (Tittel, Brendel, Zbinden, \& Gisin, 1998) and Austria (Weihs, Jennewein, Simon, Weinfurter, \& Zeilinger, 1998). More recent experiments again challenged non-contextuality (Lapkiewicz, Li, Schaeff, Langford, Ramelow, Wiesniak, \& Zeilinger, 2011, Manning, Khakimov, Dall, \& Truscott, 2015). Commenting on them, physicist Anton Zeilinger has been quoted as saying that "there is no sense in assuming that what we do not measure about a system 
has [an independent] reality" (Ananthaswamy, 2011). Finally, Dutch researchers (Hensen et al., 2015) and a large international collaboration (The BIG Bell Test Collaboration, 2018) successfully performed tests closing all potential loopholes and definitively proving quantum theory correct.

The only way out for the adherents of non-contextuality is to speculate about the existence of hidden physical properties 'smeared out' across spacetime. It turns out, however, that certain predictions of quantum theory are incompatible with non-contextuality even for a large and important class of such speculations (Leggett, 2003). Experiments have now confirmed these predictions (Gröblacher, Paterek, Kaltenbaek, Brukner, Zukowski, Aspelmeyer, \& Aeilinger, 2007; Romero, Leach, Jack, Barnett, Padgett, \& Franke-Arnold, 2010) with results so significant that the science press has felt compelled to pronounce, "Quantum physics says goodbye to reality" (Cartwright, 2007).

The surviving interpretation of QM that could, in principle, still preserve non-contextuality is Bohmian Mechanics (Bohm, 1952a, 1952b). Alas, this interpretation is plagued by a number of other problems. For instance, unlike regular QM with its Quantum Field Theory extensions, Bohmian Mechanics has no relativistic version. Physicists Raymond Streater and Luboš Motl have reviewed other compelling technical arguments against Bohmian Mechanics (Motl, 2009; Streater, 2007, pp. 103-112). Finally, recent experiments have reportedly refuted the interpretation empirically (Wolchover, 2018).

Admittedly, there is still polemic surrounding not only Bohmian Mechanics but also the experimental results that refute noncontextuality. It is nonetheless fair to say that never before has the idea of a real physical world, independent of observation, looked so precarious. Non-contextuality, if not dead, is in serious trouble.

Consequently, it seems entirely reasonable to claim that, as far as QM is concerned, the physical world people ordinarily experience is indeed akin to an 'illusion.' Prior to being observed, physical quantities are only potentials - modeled by waves of probabilities-as opposed to defined existences.

\section{Personal Physical Realities}

The authors criticized the assertion by Deepak Chopra that "the physical world, including our bodies, is a response of the observer. We cre- 
ate our bodies as we create the experience of our world" (Chopra, 1993, p. 5; Mroczkowski \& Malozemoff, 2019, p. 144). Chopra went on to acknowledge that "these are vast assumptions, the makings of a new reality, yet all are grounded in the discoveries of quantum physics made almost a hundred years ago" (Chopra, 1993, p. 7; Mroczkowski \& Malozemoff, 2019, p. 144). So, once again, the question remains whether grounding - not proof, not irrefutable evidence, just grounding for this claim of Chopra's-exists within QM.

As the authors acknowledged, QM has many different metaphysical interpretations. There is no consensus in physics regarding which interpretation is more likely, let alone true. But one of the more sober, parsimonious, and flat-out honest interpretations is Carlo Rovelli's (1996) Relational Quantum Mechanics (RQM). According to RQM, there are no absolute - that is, observer-independent-physical quantities. Instead, all physical quantities - the entire physical world-are relative to the observer in a way analogous to motion.

Rovelli (1996) summarized RQM thus:

[Because] different observers give different accounts of the same sequence of events, ... each quantum mechanical description has to be understood as relative to a particular observer. Thus, a quantum mechanical description of a certain system (state and/or values of physical quantities) cannot be taken as an "absolute" (observer-independent) description of reality, but rather as a formalization, or codification, of properties of a system relative to a given observer. (p. 1648)

The implication is that each person, as an individual observer, "inhabits' one's own physical world, as defined by the context of one's own observations. This assertion comes very close to the notion, suggested by Chopra, that each person lives in a physical reality created in response to one's own observations.

However, a reader might inquire whether RQM is true. Any definite answer to this question would overlook the morass of unending discord that prevails in the field of foundations of physics. Nevertheless, a very recent and significant experimental result has arguably proven the central and defining point of RQM: that the physical world is, indeed, relative to the observer in a way analogous to motion (Proietti et al., 2019; see also Emerging Technology from the arXiv, 2019).

Therefore, in view of the current state of play in QM, Chopra's statements-albeit speculative-are neither crazy nor ungrounded in QM. Counterintuitive as it may sound, the idea of relative physical worlds can even be reconciled with the experience that all people share 
a common environment. I address this idea more fully in Chapter 6 of my latest book, The Idea of the World (Kastrup, 2019, pp. 93-122).

\section{Choice and Randomness}

The authors criticized popular writers who suggested that intention may directly influence the physical world's transition from potentials to defined existences-that is, the so-called 'collapse of the wave function' somehow associated with an act of observation. The authors reasoned that, according to QM, collapse produces random outcomes, thereby "preventing a person from choosing or intending a particular desirable outcome" (Mroczkowski \& Malozemoff, 2019, p. 137).

I believe the authors' reasoning here is flawed. First, it is important to consider that randomness is a highly ambiguous concept: Whereas it is defined as the absence of recognizable patterns or biases-there being formal randomness tests to verify whether this is the casea truly random process can, theoretically, produce any pattern. The chance of finding a pattern in a truly random process may be small, but it isn't zero. Indeed, because it basically consists of an acknowledgment of causal ignorance, randomness is an extraordinarily accommodating notion.

Given this point, insisting that a process is random doesn't actually exclude any outcome whatsoever. It is physically coherent-whether plausible or not-that intention may indeed influence collapse outcomes without violating quantum theory. To argue that this cannot be the case merely because QM does not positively predict such an effect begs the question: The point in contention is precisely that there may be natural agencies or organizing principles that current science still fails to recognize.

Moreover, the randomness of wave function collapse is defined on the basis of a series of repeated observations of the same quantum system. For instance, if one measures the spin of an electron along a certain direction, the result will be either $1 / 2$ or $-1 / 2$. If one then resets the experiment-ensuring that all initial conditions are the same as before-and redoes the measurement, again the result will be either $1 / 2$ or $-1 / 2$. A series of such measurements will produce a string of numbers. It is the string that should meet randomness criteria. But a technically random string, of course, does not preclude the possibility that individual measurements within it can be influenced by intention in a way that may not be noticeable in the overall string. And even if an 
overall statistical bias is noticeable, no skeptic will raise an eyebrow as, theoretically, random processes can-as argued above-produce any conceivable pattern by mere chance.

\section{Synchronicity}

Quantum predictions hold only at a statistical level. The outcomes of individual measurements-that is, individual observations or events-are non-deterministic and unpredictable; quantum theory enforces no result whatsoever at the level of individual outcomes. It is this causally undetermined space that psychiatrist Carl Jung and Nobel Prize Laureate physicist Wolfgang Pauli populated with their notion of 'synchronicity': acausal meaningful coincidences that allegedly reflect archetypal patterns underlying not only the human psyche, but also the physical world at large (Jung, 1985, Jung \& Pauli, 2001).

The authors denied "that something about quantum theory may explain the serial coincidences that underlie synchronicity" (Mroczkowski \& Malozemoff, 2019, p. 150). I believe this statement, although strictly correct, is misleading in that it sets up a straw man. The point is not that QM positively accounts for synchronicities; the point is that-unlike classical physics-QM leaves space open for synchronicities. Indeed, according to QM, at its most fundamental level nature is not deterministic; there is no causal necessity enforced at that level. This notion opens the door to other organizing principles still unknown to science.

The authors repeatedly argued that wave function collapse leads to random outcomes. But this seeming randomness does not contradict synchronicity either: Regarding the latter, theorists have postulated that nature organizes itself according to global archetypal patterns. These global patterns can be easily reconciled with apparent randomness at the level of individual quantum events, as I illustrate with the following analogy.

Imagine that you toss three dice on a table, multiple times. After each toss, each individual die randomly displays a number from one to six. In other words, the behavior of each die is seemingly random from toss to toss. But now imagine that when you look at all three dice together, after every toss, you realize that either they all display an even number or they all display an odd number. This is a simple hypothetical example of a global, synchronistic pattern that can occur even when the individual constituent events, considered in isolation, 
meet randomness criteria. In Jung's words, "Within the randomness of the throwing of the dice, a 'psychic' orderedness comes into being" (Jung \& Pauli, 2001, p. 62).

If this kind of global synchronistic alignment were to happen across quantum events in the world at large, physicists would be none the wiser. For although they can test individual events in the laboratory and verify that, when taken in isolation, the events are random, they wouldn't be able to discern a global pattern within the complexity of the physical world at large; there are just too many 'dice' to look at under controlled laboratory conditions.

The relationship between synchronicity and QM, which I articulated above, has been vouchsafed by Pauli himself. After reviewing the final draft of Jung's synchronicity essay, Pauli wrote: "I . . f found that ... from the standpoint of modern physics, [the essay] is now unassailable" (Jung \& Pauli, 2001, p. 71, emphasis added).

\section{Emptiness}

Many popular writers have highlighted the fact that, when looked at closely, matter reveals itself to be mostly empty space. If one considers the total volume of an atom and compares it to the aggregate volume of its constituent mass-containing subatomic particles-such as quarks and leptons-one realizes that the atom is indeed mostly empty. The authors, however, argued that such a conclusion "bears no relation to modern quantum physics" because "the wave function [of the subatomic particles] fills space" (Mroczkowski \& Malozemoff, 2019, p. 144).

I believe the authors' argument here is flawed in more ways than one. First, they seem to have implicitly taken for granted that the wave function is ontic-that it corresponds to an objectively existing physical entity smeared out across space. There is certainly no consensus in physics that this is the case. Many physicists maintain, instead, that the wave function is merely epistemic - that it merely captures the extent of human knowledge regarding nature's future behavior. If the latter position is true, then there is nothing objectively real that "fills space" inside an atom.

Be that as it may, when one says that an atom is 'mostly empty' one is referring to the fact that most of the space in the atom contains no mass. A well-illuminated vacuum is still considered empty, in that photons have no mass. Similarly, a vacuum filled with electromagnetic fields is still empty, for fields-abstract mathematical tools-do not count as 'occupants' of space as far as the popular intuition about 
'emptiness' is concerned. Now, because mass is a measurable physical quantity-an 'observable'-one can speak of its existence only after wave function collapse - or whatever passes for collapse, because even that phenomenon is not consensus in physics today. What then remains is a set of mass-containing subatomic particles that, indeed, occupy but a tiny fraction of the atom's total volume.

There is just no denying that 20th-century subatomic physics has ushered in an understanding that contradicts popular intuitions about the solidity of matter. These intuitions are a throwback to outdated Greek atomist views. In this context, I believe it to be valid that popular writers point out to their audiences that, contrary to vulgar assumptions, matter indeed is 'mostly empty space.'

\section{Consciousness as the Agency of Collapse}

The authors criticized the notion that consciousness might be the agency behind the transition of the physical world, upon observation, from mere potentialities to defined physical quantities. They said, "this interpretation has not been proven and is not generally accepted by quantum physicists" (Mroczkowski \& Malozemoff, 2019, p. 138). Although strictly correct, such statement also sets up a straw man: No interpretation of QM has been proven or generally accepted by quantum physicists. I do not believe this state of affairs should stop all authors from ever alluding to, or speculating about, the implications of QM.

If consciousness does not cause wave function collapse-or whatever passes for collapse-then it follows that an inanimate entity of some sort must be responsible for it. Yet, the claim that inanimate objects-such as electronic detectors-can perform quantum mechanical measurements is fundamentally problematic, because the partitioning of the world into discrete inanimate objects is merely nominal to begin with. Is a rock integral to the mountain it helps constitute? If so, does it become a separate object merely by virtue of its getting detached from the mountain? And if so, does it then perform a quantum measurement-that is, an observation that causes collapse of the wave function-each time it comes back in contact with the mountain as it bounces down the slope? Brief contemplation of these questions shows that the boundaries of a detector are arbitrary.

Indeed, as John von Neumann (2018) first argued, when two inanimate objects interact they simply become quantum mechanically entangled with one another-that is, they become united in such a 
way that the behavior of one becomes inextricably linked to the behavior of the other-but no actual measurement is performed. As such, the inanimate world is a unitary, indivisible physical system governed by QM. There are no detectors performing measurements; there is only the one inanimate world. In the words of Erich Joos, "because of the non-local properties of quantum states, a consistent description of some phenomenon in quantum terms must finally include the entire universe" (Joos, 2006, p. 71).

Let me use a concrete example to be more specific. In the well-known double-slit experiment, electrons are shot through two tiny slits. When they are observed at the slits, the electrons behave as defined individual particles. But when observed only after they have passed through the slits, the 'electrons' behave as superposed potentialities. In 1998, researchers at the Weizmann Institute in Israel showed that, when detectors are placed at the slits, the electrons behave as defined individual particles (Buks, Schuster, Heiblum, Mahalu, \& Umansky 1998). At first sight, this result may seem to indicate that measurement does not require a conscious observer.

However, the output of the detectors becomes known only when it is consciously observed by a person. The hypothesis of a measurement before this conscious observation lacks compelling theoretical and empirical grounding. After all, QM offers no reason why the whole system-electrons, slits, and detectors combined-should not be in an entangled superposition before and until someone looks at the detectors' output (von Neumann, 2018). Its condition simply cannot be known. Because people cannot abstract themselves out of their knowledge, they cannot know that detectors actually perform measurements and cause wave function collapse.

Consequently, as far as people can know, before it is represented through conscious perception the world consists of a unitary superposition of potentialities. This superposition-indivisible, as quantum entanglement prevents elements of the superposition from being describable separately from one another-is incompatible with the existence of individual, separate objects and events with defined properties.

\section{Decoherence}

The authors maintained that a quantum phenomenon called 'decoherence' "is responsible for [destroying] most unique quantum interference effects, and this decoherence always happens before any conscious observation" (Mroczkowski \& Malozemoff, 2019, p. 138). They seemed 
to suggest that decoherence obviates the postulate that consciousness is the agency of collapse. In other words, the suggestion seems to have been that decoherence alone already explains the transition of a quantum system from mere potentialities to defined physical quantities. This, however, is a well-known fallacy.

If one takes for granted the existence of a macroscopic environment consisting of defined physical quantities-that is, a classical environment-from which a microscopic quantum system in a superposition is initially isolated, then it is true that any contact with the environment will destroy the superposition. Information about the overlapping potentialities will 'leak out' and become dispersed in the surrounding environment.

The problem, however, is that decoherence cannot explain how the state of the surrounding environment became defined - that is, classical - to begin with, so it doesn't solve the measurement problem or rule out the role of consciousness. As Wojciech Zurek-one of the fathers of decoherence-acknowledged,

An exhaustive answer to [the question of why we perceive a classical world instead of superposed potentialities] would undoubtedly have to involve a model of "consciousness," since what we are really asking concerns our (observers) impression that "we are conscious" of just one of the alternatives. (Zurek, 1994, p. 29)

As Joos (2006) pointed out, "the effects of decoherence just look like collapse" (p. 77). Indeed, in an essay dedicated to highlighting the role of decoherence in the emergence of a classical world from a quantum substrate, Joos ultimately concluded that some form of either wave function collapse or parallel universes is still needed (p. 75). Decoherence alone will not do.

In conclusion, decoherence does not obviate or preclude the possibility that consciousness is the agency behind collapse.

\section{Microscopic Versus Macroscopic}

Another argument line frequently repeated by the authors is that the quantum phenomena popular writers rely on occur mostly at a microscopic level. The authors seemed to imply that fundamental or metaphysical conclusions cannot be extrapolated from this microscopic realm to the macroscopic world of tables and chairs.

Although there are undeniable operational differences between the behavior of the world of tables and chairs and that of isolated micro- 
scopic quantum systems, these differences cannot be fundamental; they must, instead, be merely epiphenomenal. After all, the world is quantum, for macroscopic objects and events are just compound results of microscopic dynamics. To quote Joos (2006) once again,

[A] method for sweeping the interpretive problems under the carpet is simply to assume, or rather postulate, that quantum theory is only a theory of micro-objects, whereas in the macroscopic realm per decree (or should I say wishful thinking?) a classical description has to be valid. Such an approach leads to the endlessly discussed paradoxes of quantum theory. These paradoxes only arise because this particular approach is conceptually inconsistent ... In addition, micro- and macro-objects are so strongly dynamically coupled that we do not even know where the boundary between the two supposed realms could possibly be found. For these reasons it seems obvious that there is no boundary. (pp. 74-75; emphasis added)

He went on to say, "whichever interpretation [of QM] one prefers, the classical world view has been ruled out" (Joos, 2006, p. 76). It is this understanding that motivates popular writers to speculate about what new degrees of natural freedom may open up, in the macroscopic world, when the implications of QM are considered. There is nothing wrong-at least in principle-with this extrapolation, for there is no actual boundary between the microscopic and the macroscopic. The distinction between the two is arbitrary, nominal, motivated by convenience, and purely epistemic.

\section{Superluminal Information Transfer}

The authors frequently alluded to the "no-communication theorem' of quantum information theory to emphasize that quantum entanglement-despite its "spooky action at a distance"-cannot be used for faster-than-light information transfer. This conclusion is, of course, entirely correct. The problem is that the authors seemed to set up yet another straw man by implying that popular writers have relied on superluminal communication to account for psi phenomena. In this regard, they singled out Pim van Lommel's allusion to the notion of nonlocal consciousness.

The straw man here is as follows: The very idea of nonlocal consciousness entails that reality is fundamentally one and, as such, communication is obviated to begin with. In the words of Jonathan Schaffer (2010), "physically, there is good evidence that the cosmos forms an entangled system and good reason to treat entangled systems as irreducible wholes (p. 32, emphasis added). 
Therefore, at the most fundamental level of reality-the level in which psi phenomena allegedly occur-there is no need for information transfer to begin with. In the altered state of consciousness neardeath experiencers find themselves in, they are ostensibly one with all existence, and so the information in question is already 'in them,' so to speak. Nothing needs to be communicated from one place to another because the information is, ex hypothesi, already 'everywhere.' The allusion here is rather to something akin to Bohm's (1980) 'implicate order' than to information transfer, superluminal or otherwise.

Notice that I am not necessarily arguing for, or defending, psi, for I am not familiar enough with the subject to take an informed position either way. I am just pointing out that the argument for psi some popular writers make, though certainly reliant on the implications of QM, does not-contrary to what the authors claimed-necessarily entail superluminal communication. The allusion to entanglement is meant to underpin the possibility that the entire cosmos is fundamentally a unitary whole, not necessarily an appeal to information transfer through entanglement.

\section{Commentary}

A recurring theme in the authors' argumentation is the claim that certain views - particularly those related to foundations of physics-are not generally accepted by physicists, are controversial, disputed, and so forth. The repeated suggestion is that, unless the physics community has reached consensus regarding a certain position, nobody else should speculate about or around it. The problem, of course, is that there is no consensus regarding any position when it comes to interpretations of QM, not only those the authors criticized. Therefore, if the authors were to have it their way, all popular debate regarding the implications of QM would cease.

I do not believe this outcome would be constructive. Although there is no consensus about what is the case, there is sufficient clarity and confidence about some very important things that-physicists already know-are not the case: Naïve local-realism has been categorically refuted, and this alone has tremendous repercussions in nearly every field of human activity. This is the elephant in the room. I do not believe that authors should close their eyes to it until physicists and philosophers have reached consensus about an alternative; I do not believe that popular writers in the fields of health care and parapsychology - to name only two-should pretend that business can continue as usual, as if naïve local-realism were true. 
Although physicists are the authority when it comes to models of nature's behavior, they don't own their results. The discoveries of QM reveal the inner workings of nature and, as such, belong to $e v$ eryone, for humans are all natural beings born from, and into, this universe. As such, people are all equally entitled-perhaps even morally required - to integrate these discoveries into their meditations about life, the universe, and everything; even-to the horror of the self-appointed skeptical police-those popular writers in the fields of health care and parapsychology.

Moreover, wild-and often ungrounded-speculation isn't a privilege of non-physicists. Today, physics itself is indulging in the most farfetched feast of speculations ever concocted by the human mind: multiple different types of parallel universes, each type potentially comprising a multi-dimensional infinity of such universes; 10 spatial dimensions, many of which are supposedly curled up into tight little knots of extraordinary topological complexity; widely conflicting views about the nature of time, such as that time does not actually exist, that time is precisely the only thing that in fact exists (space being illusory), and that time exists but isn't fundamental, emerging instead from microscopic quantum processes; the accommodation of complete unknowns by mere labeling, such as the notions of dark matter and dark energy; widely differing views regarding the origin and early evolution of the universe; and the list goes on. Given all these seriously discussed hypotheses, it is difficult for physicists to take the moral high ground and criticize non-physicists based merely on the fact that the latter are engaging in physical speculation. Compared to the conjectures of many professional physicists, allusions to quantum phenomena in health care and parapsychological literature sound rather moderate and conservative.

I acknowledge that this is not the authors' intended point or the spirit of their criticism. For them the problem is not per se that popular writers are engaging in physical speculation, but that these writers may be trying to misappropriate the authority of physics so to pass false or implausible claims for scientific fact. This kind of misappropriation is doubtlessly pernicious, dangerous, and I condemn it in the strongest terms.

However, as I have argued in this response, I do not believe that this really is what the popular writers that the authors singled out were doing. What these writers stated in their works seems to me to be, by and large, reasonable enough-if poorly worded—given recent results from physics. 
Although attempting to do something doubtlessly valid and important, I suspect the authors, by overshooting the mark, may have contributed to the very confusion they were trying to combat. This outcome is unfortunate-but shouldn't stop efforts to separate wheat from chaff and bring some clarity to the reigning confusion around the foundations of quantum mechanics.

\section{References}

Ananthaswamy, A. (2011, June 22). Quantum magic trick shows reality is what you make it. New Scientist. Retrieved from https://www.newscientist.com /article/dn20600-quantum-magic-trick-shows-reality-is-what-you-make-it/

Aspect, A., Dalibard, J., \& Roger, G. (1982). Experimental test of Bell's inequalities using time-varying analyzers. Physical Review Letters, 49(25), 1804-1807.

Aspect, A., Grangier, P., \& Roger, G. (1981). Experimental tests of realistic local theories via Bell's theorem. Physical Review Letters, 47(7), 460-463.

Aspect, A., Grangier, P., \& Roger, G. (1982). Experimental realization of EinsteinPodolsky-Rosen-Bohm gedankenexperiment: A new violation of Bell's inequalities. Physical Review Letters, 49(2), 91-94.

Bohm, D. (1952a). A suggested interpretation of the quantum theory in terms of "hidden" variables. I. Physical Review, 85, 166-179.

Bohm, D. (1952b). A suggested interpretation of the quantum theory in terms of "hidden" variables. II. Physical Review, 85, 180-193.

Bohm, D. (1980). Wholeness and the implicate order. Londong, England, UK: Routledge.

Buks, E., Schuster, R., Heiblum, M., Mahalu, D., \& Umansky, V. (1998). Dephasing in electron interference by a 'which-path' detector. Nature, 391, 871-874.

Cartwright, J. (2007, April 20). Quantum physics says goodbye to reality. IOP Physics World. Retrieved from https://physicsworld.com/a/quantum-physics -says-goodbye-to-reality/.

Emerging Technology from the arXiv. (2019, March 12). A quantum experiment suggests there's no such thing as objective reality. MIT Technology Review. Retrieved from https:/www.technologyreview.com/s/613092/a-quantum -experiment-suggests-theres-no-such-thing-as-objective-reality/

Gröblacher, S., Paterek, T., Kaltenbaek, R., Brukner, C., Zukowski, M., Aspelmeyer, M., \& Aeilinger, A. (2007). An experimental test of non-local realism. Nature, 446, 871-875.

Hensen, B., Bernien, H., Dreau, A. E., Reiserer, A., Kalb, N., Blok, M. S. . . . Hanson, R. ( 2015). Loophole-free Bell inequality violation using electron spins separated by 1.3 kilometres. Nature, $526,682-686$.

Joos, E. (2006). The emergence of classicality from quantum theory. In P. Clayton \& P. Davies (Eds), The re-emergence of emergence: The emergentist hypothesis from science to religion. Oxford, England, UK: Oxford University Press.

Jung, C. G. (1985). Synchronicity: An acausal connecting principle. London, England, UK: Routledge.

Jung, C. G., \& Pauli, W. (2001). In C. A. Meier (Ed.), Atom and archetype: The Pauli/Jung letters 1932-1958. London, England, UK: Routledge. 
Kastrup, B. (2019). The idea of the world: A multi-disciplinary argument for the mental nature of reality. Winchester, England, UK: Iff Books.

Lapkiewicz, R., Li, P., Schaeff, C., Langford, N. K., Ramelow, S. Wiesniak, M., \& Zeilinger, A. (2011). Experimental non-classicality of an indivisible quantum system. Nature, 474, 490-493.

Leggett, A. N. (2003). Nonlocal hidden-variable theories and quantum mechanics: An incompatibility theorem. Foundations of Physics, 33(10), 1469-1493.

Manning, A. G., Khakimov, R. I., Dall, R. G., \& Truscott, A. G. (2015). Wheeler's delayed-choice gedanken experiment with a single atom. Nature Physics, 11, 539-542.

Motl, L. (2009, January 23). Bohmists \& segregation of primitive and contextual observables. The Reference Frame. Retrieved from https://motls.blogspot .com/2009/01/bohmists-segregation-of-primitive-and.html.

Mroczkowski, J. A., \& Malozemoff, A. P. (2019). Quantum misuse in psychic literature. Journal of Near-Death Studies, 37, 131-154. doi:10.17514/JNDS-2019 -37-3-p131-154.

Proietti, M., Pickston, A., Graffitti, F., Barrow, P., Kundys, D., Branciard, C., . . Fedrizzi, A. (2019). Experimental rejection of observer-independence in the quantum world. arXiv:1902.05080 [quant-ph], 1-10. Retrieved from https:// arxiv.org/abs/1902.05080.

Romero, J., Leach, J., Jack, B., Barnett, S. M., Padgett, M. J., \& Franke-Arnold, S. (2010). Violation of Leggett inequalities in orbital angular momentum subspaces. New Journal of Physics, 12, 123007.

Rovelli, C. (1996). Relational quantum mechanics. International Journal of Theoretical Physics, 35(8), 1637-1678.

Schaffer, J. (2010). Monism: The priority of the whole. Philosophical Review, 119(1), 31-76.

Streater, R. F. (2007). Lost causes in and beyond physics. Berlin, Germany: Springer-Verlag.

The BIG Bell Test Collaboration, (2018). Challenging local realism with human choices. Nature, 557, 212-216.

Tittel, W., Brendel, J., Zbinden, H., \& Gisin, N. (1998). Violation of Bell inequalities by photons more than $10 \mathrm{~km}$ apart. Physical Review Letters, 81(17), 3563-3566.

von Neumann, J. (2018). Mathematical foundations of quantum mechanics: New edition. Princeton, NJ: Princeton University Press.

Weihs, G., Jennewein, T., Simon, C., Weinfurter, H., \& Zeilinger, A. (1998). Violation of Bell's inequality under strict Einstein locality conditions. Physical Review Letters, 81(23), 5039-5043.

Wolchover, N. (2018, October 11). Famous experiment dooms alternative to quantum weirdness. Quanta Magazine. Retrieved from https://www.quanta magazine.org/famous-experiment-dooms-pilot-wave-alternative-to-quantum -weirdness-20181011/.

Zurek, W. H. (1994). Preferred observables, predictability, classicality, and the environment-induced decoherence. arXiv:gr-qc/9402011v1. Retrieved from https://arxiv. org/abs/gr-qc/9402011. 